\title{
Proposta de classificação dos coeficientes de variação em relação à produtividade e altura da planta de soja(1)
}

\author{
Claudio Guilherme Portela de Carvalho ${ }^{(2)}$, Carlos Alberto Arrabal Arias ${ }^{(3)}$, José Francisco Ferraz de Toledo ${ }^{(3)}$, \\ Leones Alves de Almeida ${ }^{(3)}$, Romeu Afonso de Souza Kiihl(3), Marcelo Fernandes de Oliveira ${ }^{(3)}$, \\ Dario Minoru Hiromoto(4) e Claudio Takeda ${ }^{(4)}$
}

Resumo - O objetivo deste trabalho foi definir classificações de coeficientes de variação para produtividade e altura da planta de soja. Os dados foram obtidos de ensaios intermediários e finais realizados nos estados do Paraná e do Mato Grosso. Foram realizadas classificações distintas para cada localização e ciclo reprodutivo. Considerando-se a média e o desvio-padrão dos coeficientes de variação obtidos das análises de variância dos ensaios, os coeficientes foram classificados como baixo, médio, alto e muito alto. Uma classificação adicional foi feita utilizando a mediana e o pseudo-sigma, em substituição à média e ao desvio-padrão, respectivamente. A classificação dos coeficientes de variação dependeu do caráter e da localização, mas não variou muito em razão do ciclo reprodutivo. Os critérios adotados (média e desvio-padrão ou mediana e pseudo-sigma) foram semelhantes (independentemente da distribuição dos coeficientes de variação) e satisfatórios para determinar a precisão experimental. O limite máximo de coeficiente de variação aceitável para produtividade é de $16 \%$ e para altura da planta é de $12 \%$.

Termos para indexação: Glycine max, componente de rendimento, análise estatística.

\section{Proposal to categorize coefficients of variation for yield and plant height in soybean}

\begin{abstract}
The objective of this work was to define categories for the coefficients of variation obtained in soybean plant height and yield. The experimental data were obtained in intermediary and final (regional) adaptation trials carried out in the States of Paraná and Mato Grosso. Different categories were established for each State and maturity group. Taking the mean and the standard deviation of the coefficients of variation obtained from the analyses of variance of the experiments, the coefficients of variation were classified as low, average, high and very high. An additional classification was made using the median and pseudo-sigma as substitutes for mean and standard deviation, respectively. The classification for the coefficients of variation depended on the trait and the location but did not vary greatly for each maturity group. The adopted criteria (mean and standard deviation or median and pseudo-sigma) were similar (regardless of the coefficient of variation distribution), and satisfactory in determining the experimental accuracy. The maximum acceptable limits for coefficient of variation are $16 \%$ and $12 \%$ for yield and plant height, respectively.
\end{abstract}

Index terms: Glycine max, yield components, statistical analysis.

(1) Aceito para publicação em 14 de novembro de 2002. Parcialmente financiado pelo CNPq.

${ }^{(2)}$ Embrapa-Centro Nacional de Pesquisa de Soja (CNPSo), Caixa Postal 231, CEP 86001-970 Londrina, PR. Bolsista do CNPq. E-mail: cportela@cnpso.embrapa.br

(3)Embrapa-CNPSo. E-mail: arias@cnpso.embrapa.br, toledo@cnpso.embrapa.br, leones@cnpso.embrapa.br, romeu@cnpso.embrapa.br, marcelo@cnpso.embrapa.br

(4)Fundação de Apoio à Pesquisa Agropecuária de Mato Grosso, Caixa Postal 79, CEP 78705-040 Rondonópolis, MT. E-mail: fundacao@fundacaomt.com.br

\section{Introdução}

O coeficiente de variação $(\mathrm{CV})$ obtido da análise de variância de um ensaio experimental indica o grau de precisão do experimento. Com base nos coeficientes estimados nos ensaios realizados no campo, Pimentel-Gomes (1985) classificou-os como baixos, quando inferiores a $10 \%$; médios, quando de $10 \%$ a $20 \%$; altos, quando de $20 \%$ a $30 \%$, e muito altos, quando superiores a $30 \%$. Contudo, essa classifica- 
ção é muito abrangente e não leva em consideração as particularidades da cultura estudada, e, principalmente, não faz distinção quanto a natureza do caráter avaliado (Garcia, 1989; Scapim et al., 1995; Costa et al., 2002). Além disso, essa classificação pode variar dependendo das condições edafoclimáticas ou ciclo reprodutivo da cultura (Scapim et al., 1995).

Pressupondo distribuição normal e considerando a média (m) e o desvio-padrão (DP) dos coeficientes de variação, estimados nas análises de variância de ensaios experimentais, relativos às espécies de Eucaliptus e Pinus, Garcia (1989) propôs uma nova classificação dos CV, específica para a realidade florestal. Para um determinado caráter, os CV foram classificados como baixo $[\mathrm{CV} \leq(\mathrm{m}-1 \mathrm{DP})]$, médio $[(m-1 \mathrm{DP})<\mathrm{CV} \leq(\mathrm{m}+1 \mathrm{DP})]$, alto $[(\mathrm{m}+$ $1 \mathrm{DP})<\mathrm{CV} \leq(\mathrm{m}+2 \mathrm{DP})]$ e muito alto $[\mathrm{CV}>$ $(\mathrm{m}+2 \mathrm{DP})]$. Esses critérios de classificação foram também utilizados em milho (Scapim et al., 1995). Quando os CV não apresentam distribuição normal, Costa et al. (2002) sugeriram a utilização das estatísticas mediana e pseudo-sigma, em substituição à média e ao desvio-padrão, respectivamente. Segundo esses autores, quando há normalidade, essas duas metodologias são equivalentes.

Num programa de melhoramento genético, a classificação de CV pode ser útil, por exemplo, para informar a qualidade experimental de ensaios intermediários e finais dessa cultura. Nesses ensaios, um conjunto de caracteres é mensurado para auxiliar o melhorista na descrição e indicação de novas cultivares. Na soja [Glycine max (L.) Merrill], os caracteres quantitativos importantes avaliados nos programas de melhoramento são produtividade, teor de óleo, teor de proteína, altura de planta, dias para maturação, dias para floração, entre outros.

Na elaboração do VCU (valor de cultivo e uso) para o registro de novas cultivares de soja, deve-se considerar apenas os ensaios cujos $\mathrm{CV}$ de produtividade forem inferiores a 20\%. Esse valor máximo é estabelecido pelo Ministério da Agricultura, Pecuária e Abastecimento para a soja e demais culturas, cujas cultivares podem ser registradas (Brasil, 2001). Na classificação de Pimentel-Gomes (1985), coeficientes de variação inferiores a $20 \%$ são con- siderados baixos ou médios. Contudo, a adoção de um valor máximo de $\mathrm{CV}$ para produtividade, específico para uma cultura, pode ser mais adequada. Além disso, apesar de nenhum valor máximo de CV ser estabelecido, uma classificação específica para os demais caracteres de soja auxiliaria também na melhor caracterização de novas cultivares.

O objetivo deste trabalho foi definir classificações de coeficientes de variação para produtividade e altura da planta de soja.

\section{Material e Métodos}

Foram analisados os dados dos ensaios intermediários e finais de linhagens de soja, conduzidos em diversos locais dos estados do Paraná e do Mato Grosso, pela Embrapa-Centro Nacional de Pesquisa de Soja e parceiros.

Nos ensaios realizados no Paraná, entre 1989/1990 e 1998/1999, incluíram-se linhagens dos grupos de maturação precoce (110 a 115 dias), semiprecoce (116 a 125 dias) e médio (126 a 137 dias). No Mato Grosso, os ensaios realizaram-se entre 1994/1995 e 1999/2000. Neste Estado, os ciclos semiprecoce e médio são denominados médio e tardio, respectivamente. Em cada ano, diferentes linhagens foram testadas nas duas localizações e as de melhor desempenho agronômico permaneciam em avaliação por dois ou mais anos consecutivos ao teste. Nos ensaios intermediários, essa permanência era de apenas um ano.

A rede experimental abrangeu locais que foram escolhidos pela diversidade e representatividade dos diferentes ecossistemas do Paraná e do Mato Grosso. A data de semeadura variou com o ano e local da avaliação, prevalecendo entre 15 e 25 de novembro. O delineamento experimental foi o de blocos completos casualizados, com três repetições, nos ensaios intermediários, e quatro repetições nos ensaios finais. Cada parcela era constituída de quatro linhas de cinco metros espaçadas de 0,45 a $0,50 \mathrm{~m}$. Foram colhidas apenas as duas linhas centrais de cada parcela, descartando-se $0,5 \mathrm{~m}$ em cada extremidade, resultando em área útil de 3,6 a 4,0 m². A densidade foi de aproximadamente 40 plantas $/ \mathrm{m}^{2}$. Adubação, controle fitossanitário, capina e demais tratos culturais foram realizados de modo a manter as plantas sob condições ótimas de crescimento e desenvolvimento. 
Os caracteres avaliados foram produtividade $(\mathrm{kg} / \mathrm{ha})$, teor de óleo (\%), teor de proteína (\%), altura da planta $(\mathrm{cm})$, dias para maturação e floração e resistência a algumas das principais doenças da cultura. Apenas a produtividade e a altura da planta foram avaliadas em todas as repetições e as classificações dos seus coeficientes de variação, estimados a partir das análises de variância dos ensaios, foram feitas com base nos métodos de Garcia (1989) e Costa et al. (2002). A existência de distribuição normal dos CV foi averiguada por meio do teste de Shapiro-Wilk (SAS Institute, 1990).

Considerando-se a média (m) e o desvio-padrão (DP) dos CV obtidos nas análises de variância, os coeficientes foram classificados, de acordo com Garcia (1989). No método de Costa et al. (2002), os intervalos de classificação são definidos similarmente ao de Garcia (1989). Contudo, m e DP são substituídos, respectivamente, pela mediana (Md) e pelo pseudo-sigma (PS);

$\mathrm{Md}=\left(\mathrm{Q}_{1}+\mathrm{Q}_{3}\right) / 2$ é a mediana dos coeficientes de variação para o primeiro $\left(\mathrm{Q}_{1}\right)$ e o terceiro $\left(\mathrm{Q}_{3}\right)$ quartil, respectivamente, os quais delimitam $25 \%$ de cada extremidade da distribuição e;

PS = AI/1,35 é o pseudo-sigma (Tukey, 1977; Hoaglin et al., 1983) para a amplitude interquartílica (AI). Essa medida indica o quanto os dados estão distanciados da mediana. Segundo Costa et al. (2002), o pseudo-sigma seria o desvio-padrão que uma distribuição normal precisaria ter para produzir a mesma amplitude interquartílica da distribuição dos dados amostrais. O valor 1,35 corresponde à distância entre $\mathrm{Q}_{1}$ e $\mathrm{Q}_{3}$ na distribuição normal $(\mathrm{N} \sim(0,1))$.

As classificações dos CV foram feitas para cada caráter e de forma específica para cada localização (Paraná e Mato Grosso) e ciclo reprodutivo das linhagens. Para testar a significância dos efeitos de caráter $(i=1,2)$, localização $(j=1,2)$ e ciclo reprodutivo $(k=1,2,3)$ na magnitude dos CV, foi aplicado o teste $\mathrm{t}$ (Pimentel-Gomes, 1985). No teste do efeito de caráter foram usadas as diferenças entre médias $\left(\mathrm{m}_{\mathrm{ijk}}-\mathrm{m}_{\mathrm{i}{ }^{\prime} \mathrm{jk}}\right)$ dos $\mathrm{CV}$ dos caracteres, obtidas para uma mesma localização e grupo de maturação. No teste do efeito de localização foram usadas as diferenças entre médias $\left(\mathrm{m}_{\mathrm{ijk}}-\mathrm{m}_{\mathrm{ij}}{ }^{\mathrm{k}} \mathrm{k}\right)$ dos $\mathrm{CV}$ de um mesmo caráter, obtidas para um mesmo grupo de maturação, mas localizações diferentes. No teste do efeito de ciclo reprodutivo foram usadas as diferenças entre médias $\left(\mathrm{m}_{\mathrm{ijk}}-\mathrm{m}_{\mathrm{ijk}}\right.$ ) dos CV de um mesmo caráter, obtidas em uma mesma localização, mas em linhagens de grupos de maturação distintos.
As análises estatísticas foram feitas usando os procedimentos 'PROC UNIVARIATE' e 'PROC GLM' do módulo de estatística do SAS (SAS Institute, 1990).

\section{Resultados e Discussão}

Diferenças significativas entre médias $\left(\mathrm{m}_{\mathrm{ijk}}-\mathrm{m}_{\mathrm{i}}{ }_{\mathrm{jk}}\right)$ dos $\mathrm{CV}$ dos caracteres foram obtidas para uma mesma localização e grupo de maturação, a $0,1 \%$ de probabilidade, indicando haver efeito de caráter na magnitude dos CV (Tabelas 1 e 2). Em média, os valores de CV para produtividade foram maiores que para altura da planta. $\mathrm{O}$ efeito de localização $\left(m_{i j k}-m_{i j ' k}\right)$ também foi significativo $(\mathrm{P} \leq 0,001)$, principalmente para altura de planta. Em relação a esse caráter, valores de Mato Grosso superaram os do Paraná. Por sua vez, o efeito de ciclo reprodutivo $\left(\mathrm{m}_{\mathrm{ijk}}-\mathrm{m}_{\mathrm{ijk}}\right)$ não foi significativo $(\mathrm{P} \geq 0,05)$, com exceção de um único contraste.

Na classificação de Garcia (1989), além de m, deve ser considerado também o DP dos CV. Os DPs obtidos para produtividade nunca foram inferiores aos obtidos para altura de planta e classificações distintas foram definidas em relação a esses caracteres (Tabelas 1 e 3). Em ensaios de linhagens de mesmo ciclo de maturação, os DPs estimados em relação à altura da planta no Mato Grosso e no Paraná foram similares. Contudo, foram definidas classificações distintas quanto a altura da planta nas duas localizações, por causa das diferenças entre médias. Essa distinção associada à localização foi menos pronunciada em relação à produtividade. De modo similar à m, o DP e a classificação dos CV tenderam a não variar muito em razão do ciclo reprodutivo nas duas localizações.

Considerando a classificação com base em m e DP e ensaios de produtividade de linhagens precoces de soja semeadas no Paraná, os coeficientes de variação foram considerados baixos, $\mathrm{CV} \leq 8,2 \%$; médios, 8,2\% < CV $\leq 14,9 \%$; altos, $14,9 \%<\mathrm{CV} \leq 18,2 \%$, e muito altos $\mathrm{CV}>18,2 \%$ (Tabela 3). Com base nessa classificação, as frequiências dos CV observados nos referidos intervalos foi de $11,9 \%, 74,4 \%, 8,9 \%$ e $4,8 \%$, respectivamente (Tabela 4). Ao se adotar a classificação de Pimentel- 
Tabela 1. Teste de Shapiro-Wilk e estatísticas descritivas dos coeficientes de variação em relação à produtividade e à altura da planta de soja, obtidos dos ensaios intermediários e finais, realizados pela Embrapa-Centro Nacional de Pesquisa de Soja e parceiros, entre 1990 e 1999, nos estados do Paraná e do Mato Grosso.

\begin{tabular}{|c|c|c|c|c|c|c|c|c|}
\hline \multirow{2}{*}{$\begin{array}{l}\text { Grupo de } \\
\text { maturação }\end{array}$} & \multirow{2}{*}{$\begin{array}{l}\text { Número de } \\
\text { informações }\end{array}$} & \multirow{2}{*}{$\begin{array}{c}\text { Teste de } \\
\text { Shapiro-Wilk }\end{array}$} & \multicolumn{6}{|c|}{ Coeficiente de variação (\%) } \\
\hline & & & Média & Desvio-padrão & Mediana & Pseudo-sigma & Mínimo & Máximo \\
\hline & \multicolumn{8}{|c|}{ Paraná - Produtividade } \\
\hline Precoce & 168 & $0,912 * *$ & 11,53 & 3,32 & 11,21 & 2,93 & 5,87 & 23,96 \\
\hline Semi-precoce & 170 & $0,927 * *$ & 11,92 & 3,61 & 11,44 & 3,28 & 4,72 & 24,87 \\
\hline Médio & 171 & $0,943 * *$ & 12,26 & 3,55 & 11,88 & 3,13 & 5,80 & 25,32 \\
\hline \multicolumn{9}{|c|}{ Paraná - Altura da Planta } \\
\hline Precoce & 114 & $0,905 * *$ & 7,95 & 2,84 & 7,69 & 2,57 & 3,40 & 17,47 \\
\hline Semi-precoce & 129 & $0,954 * *$ & 7,20 & 2,25 & 7,12 & 2,02 & 2,21 & 15,17 \\
\hline Médio & 122 & $0,926 * *$ & 7,38 & 2,67 & 7,04 & 2,33 & 2,49 & 14,91 \\
\hline \multicolumn{9}{|c|}{ Mato Grosso - Produtividade } \\
\hline Precoce & 79 & $0,978^{\mathrm{ns}}$ & 12,75 & 2,85 & 12,69 & 2,68 & 6,92 & 20,75 \\
\hline Médio & 80 & $0,949 * *$ & 12,30 & 3,18 & 12,46 & 3,50 & 6,80 & 19,53 \\
\hline Tardio & 81 & $0,971^{\mathrm{ns}}$ & 12,65 & 2,96 & 12,73 & 2,49 & 7,52 & 21,89 \\
\hline & \multicolumn{8}{|c|}{ Mato Grosso - Altura da Planta } \\
\hline Precoce & 79 & $0,931 * *$ & 9,28 & 2,84 & 9,00 & 3,04 & 4,22 & 16,81 \\
\hline Médio & 81 & $0,953 *$ & 8,72 & 2,49 & 8,57 & 2,73 & 4,87 & 15,69 \\
\hline Tardio & 79 & $0,964^{\mathrm{ns}}$ & 8,71 & 2,40 & 8,54 & 2,77 & 4,45 & 14,66 \\
\hline
\end{tabular}

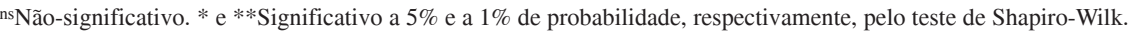

Tabela 2. Comparação entre médias dos coeficientes de variação de caracteres de soja $[i=1$ (produtividade), 2 (altura de planta)], em diferentes estados da Federação [j = 1 (Paraná), 2 (Mato Grosso)], em diferentes ciclos reprodutivos $\left[\mathrm{k}=1\right.$ (Precoce), 2 (Médio), 3 (Tardio) ${ }^{(1)}$.

\begin{tabular}{|c|c|}
\hline Comparação de médias $\left(\mathrm{m}_{\mathrm{ijk}}\right)$ & Valor de $\mathrm{t}$ \\
\hline \multicolumn{2}{|c|}{ Caráter (produtividade $\mathrm{x}$ altura da planta) } \\
\hline $\mathrm{m}_{111}-\mathrm{m}_{211}$ & $9,69^{* * * *}$ \\
\hline $\mathrm{m}_{112}-\mathrm{m}_{212}$ & $13,86^{* * *}$ \\
\hline $\mathrm{m}_{113}-\mathrm{m}_{213}$ & $13,43^{* * * *}$ \\
\hline $\mathrm{m}_{121}-\mathrm{m}_{221}$ & $7,66^{* * * *}$ \\
\hline$m_{122}-m_{222}$ & $7,95^{* * * *}$ \\
\hline $\mathrm{m}_{123}-\mathrm{m}_{223}$ & $9,26^{* * *}$ \\
\hline \multicolumn{2}{|c|}{ Localização (Paraná x Mato Grosso) } \\
\hline $\mathrm{m}_{111}-\mathrm{m}_{121}$ & $-2,97^{* *}$ \\
\hline $\mathrm{m}_{112}-\mathrm{m}_{122}$ & $-0,84^{\mathrm{ns}}$ \\
\hline $\mathrm{m}_{113}-\mathrm{m}_{123}$ & $-0,91^{\text {ns }}$ \\
\hline $\mathrm{m}_{211}-\mathrm{m}_{221}$ & $-3,20^{* *}$ \\
\hline $\mathrm{m}_{212}-\mathrm{m}_{222}$ & $-4,46^{* * *}$ \\
\hline $\mathrm{m}_{213}-\mathrm{m}_{223}$ & $-3,66^{* * *}$ \\
\hline \multicolumn{2}{|c|}{$\begin{array}{c}\text { Ciclo reprodutivo (precoce } \mathrm{x} \text { médio) ou (precoce } \mathrm{x} \text { tardio) } \\
\text { ou (médio } \mathrm{x} \text { tardio) }\end{array}$} \\
\hline $\mathrm{m}_{111}-\mathrm{m}_{112}$ & $1,03^{\text {ns }}$ \\
\hline $\mathrm{m}_{111}-\mathrm{m}_{113}$ & $1,95^{\text {ns }}$ \\
\hline $\mathrm{m}_{112}-\mathrm{m}_{113}$ & $0,87^{\mathrm{ns}}$ \\
\hline $\mathrm{m}_{211}-\mathrm{m}_{212}$ & $2,26^{* * *}$ \\
\hline $\mathrm{m}_{211}-\mathrm{m}_{213}$ & $1,58^{\mathrm{ns}}$ \\
\hline $\mathrm{m}_{212}-\mathrm{m}_{213}$ & $0,57^{\mathrm{ns}}$ \\
\hline $\mathrm{m}_{121}-\mathrm{m}_{122}$ & $0,94^{\mathrm{ns}}$ \\
\hline $\mathrm{m}_{121}-\mathrm{m}_{123}$ & $0,22^{\text {ns }}$ \\
\hline $\mathrm{m}_{122}-\mathrm{m}_{123}$ & $-0,72^{\text {ns }}$ \\
\hline$m_{221}-m_{222}$ & $1,32^{\text {ns }}$ \\
\hline$m_{221}-m_{223}$ & $1,36^{\mathrm{ns}}$ \\
\hline $\mathrm{m}_{222}-\mathrm{m}_{223}$ & $0,03^{\text {ns }}$ \\
\hline
\end{tabular}

(1)No Paraná, os ciclos médio e tardio são denominados semiprecoce e

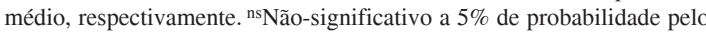
teste t. $* * \mathrm{e} * * *$ Significativo a $1 \%$ e a $0,1 \%$ de probabilidade, respectivamente, pelo teste $\mathrm{t}$
Gomes (1985), apenas 1,79\% dos CV foram considerados altos $(20 \%<\mathrm{CV} \leq 30 \%)$ e $0 \%$, muito altos $(\mathrm{CV}>30 \%)$. Esse reduzido número de ensaios com baixa precisão experimental (CV > 20\%) foi observado, também, na avaliação da produtividade de linhagens dos demais grupos de maturação, no Paraná e Mato Grosso (Tabela 4). A melhor distribuição dos CV nos diferentes níveis de classificação, quando se utiliza m e DP, foi considerada, por Scapim et al. (1995), um indício da superioridade desse critério, em relação ao de Pimentel-Gomes (1985).

Além da produtividade, resultados pouco conservadores foram obtidos em relação à altura da planta ao se utilizar o método de PimentelGomes (1985), pois nenhum coeficiente de variação associado a esse caráter foi superior a $20 \%$ e, por conseqüência, classificado como alto ou muito alto (Tabelas 1 e 4). Isto não foi verificado em relação aos componentes de rendimento de milho (Scapim et al., 1995). Nesta cultura, há boa concordância na definição dos intervalos de classificação com base em Pimentel-Gomes (1985) e em Garcia (1989), quando os caracteres, por exemplo a produtividade, são muito influenciados pelo ambiente. Mas, em relação aos menos influenciados pelo ambiente como altura de planta, o último método mostra-se mais adequado. 
Tabela 3. Intervalo dos coeficientes de variação segundo os critérios de classificação de Garcia (1989) e Costa et al. (2002), a partir dos dados de produtividade e altura da planta de soja obtidos dos ensaios intermediários e finais dessa cultura em razão do grupo de maturação (precoce, semiprecoce, médio e tardio).

\begin{tabular}{|c|c|c|c|c|}
\hline \multirow{2}{*}{$\begin{array}{l}\text { Classificação } \\
\text { dos coeficientes } \\
\text { de variação }\end{array}$} & \multicolumn{2}{|c|}{ Garcia (1989) } & \multicolumn{2}{|c|}{ Costa et al. (2002) } \\
\hline & Produtividade & Altura da planta & Produtividade & Altura da planta \\
\hline & \multicolumn{4}{|c|}{ Paraná - Precoce } \\
\hline Baixo & $\leq 8,2$ & $\leq 5,1$ & $\leq 8,3$ & $\leq 5,1$ \\
\hline Médio & $8,2-14,9$ & $5,1-10,8$ & $8,3-14,1$ & $5,1-10,3$ \\
\hline Alto & $14,9-18,2$ & $10,8-13,6$ & $14,1-17,1$ & $10,3-12,8$ \\
\hline \multirow[t]{2}{*}{ Muito alto } & $>18,2$ & $>13,6$ & $>17,1$ & $>12,8$ \\
\hline & \multicolumn{4}{|c|}{ Paraná - Semiprecoce } \\
\hline Baixo & $\leq 8,3$ & $\leq 5,0$ & $\leq 8,2$ & $\leq 5,1$ \\
\hline Médio & $8,3-15,5$ & $5,0-9,5$ & $8,2-14,7$ & $5,1-9,2$ \\
\hline Alto & $15,5-19,1$ & $9,5-11,7$ & $14,7-18,0$ & $9,2-11,2$ \\
\hline \multirow{2}{*}{ Muito alto } & $>19,1$ & $>11,7$ & $>18,0$ & $>11,2$ \\
\hline & \multicolumn{4}{|c|}{ Paraná - Médio } \\
\hline Baixo & $\leq 8,7$ & $\leq 4,7$ & $\leq 8,8$ & $\leq 4,7$ \\
\hline Médio & $8,7-15,8$ & $4,7-10,1$ & $8,8-15,0$ & $4,7-9,4$ \\
\hline Alto & $15,8-19,4$ & $10,1-12,7$ & $15,0-18,1$ & $9,4-11,7$ \\
\hline \multirow[t]{2}{*}{ Muito alto } & $>19,4$ & $>12,7$ & $>18,1$ & $>11,7$ \\
\hline & \multicolumn{4}{|c|}{ Mato Grosso - Precoce } \\
\hline Baixo & $\leq 9,9$ & $\leq 6,4$ & $\leq 10,0$ & $\leq 6,2$ \\
\hline Médio & $9,9-15,6$ & $6,4-12,0$ & $10,0-15,4$ & $6,2-12,0$ \\
\hline Alto & $15,6-18,5$ & $12,0-15,0$ & $15,4-18,0$ & $12,0-15,0$ \\
\hline \multirow{2}{*}{ Muito alto } & $>18,5$ & $>15,0$ & $>18,0$ & $>15,0$ \\
\hline & \multicolumn{4}{|c|}{ Mato Grosso - Médio } \\
\hline Baixo & $\leq 9,1$ & $\leq 6,2$ & $\leq 9,0$ & $\leq 5,8$ \\
\hline Médio & $9,1-15,5$ & $6,2-11,2$ & $9,0-16,0$ & $5,8-11,3$ \\
\hline Alto & $15,5-18,7$ & $11,2-13,7$ & $16,0-19,5$ & $11,3-14,0$ \\
\hline \multirow[t]{2}{*}{ Muito alto } & $>18,7$ & $>13,7$ & $>19,5$ & $>14,0$ \\
\hline & \multicolumn{4}{|c|}{ Mato Grosso - Tardio } \\
\hline Baixo & $\leq 9,7$ & $\leq 6,3$ & $\leq 10,2$ & $\leq 5,8$ \\
\hline Médio & $9,7-15,6$ & $6,3-11,1$ & $10,2-15,2$ & $5,8-11,3$ \\
\hline Alto & $15,6-18,6$ & $11,1-13,5$ & $15,2-17,7$ & $11,3-14,0$ \\
\hline Muito alto & $>18,6$ & $>13,5$ & $>17,7$ & $>14,0$ \\
\hline
\end{tabular}

De modo geral, os coeficientes de variação associados à produtividade e altura da planta de soja não apresentaram distribuição normal (Tabela 1). Nessas condições, Costa et al. (2002) relatam que Md e PS são medidas mais robustas que m e DP, respectivamente. Segundo esses autores, outra vantagem do uso daqueles parâmetros é possibilitar que a definição dos intervalos de classificação não dependa da distribuição dos CV. Contudo, no presente trabalho, não houve muita diferença na definição (Tabela 3) dos intervalos em relação aos caracteres de soja avaliados, mesmo nas situações de não ocorrência de normalidade dos CV (Tabela 1). Por consequiência, a importância dos efeitos de caráter, localização e ciclo reprodutivo tenderam a se manter com a substituição de m e DP, por Md e PS (Tabelas 1 e 3). Diferenças quanto a frequiências de distribuição dos CV foi encontrada ao substituir a média e desviopadrão por mediana e pseudo-sigma (Tabela 4). Isto foi verificado principalmente para a quarta classe (muito alto). Por exemplo, a frequiência de CV muito alto, no Paraná, quase dobrou ao ser realizada a substituição. Mas as diferenças nas frequiências não interferiram muito na definição dos intervalos de classificação. 
Como mencionado anteriormente, as médias (ou medianas) e os limites dos intervalos de classificação dos $\mathrm{CV}$, referentes à altura da planta, foram relativamente menores às obtidas em relação à produtividade (Tabelas 1 e 2). Isso mostra a importância do uso de uma classificação de CV específica para um caráter. Mas, apesar da classificação dos CV em relação aos caracteres (principalmente, altura de planta) da soja depender da localização avaliada, o estabelecimento de um único limite máximo aceitável para o coeficiente de variação de um caráter é possível e facilita o seu uso. Assim, adotando-se os critérios (m e DP ou Md e PS) estabelecidos nesse estudo, $\mathrm{CV}$ menores que $16 \%$ para produtividade ou $12 \%$ para altura da planta, foram sempre considerados médios ou baixos, para todos os ciclos reprodutivos das linhagens e localizações avaliadas.
O valor estimado para produtividade é inferior ao estabelecido pelo Ministério da Agricultura, Pecuária e Abastecimento $(\mathrm{CV} \leq 20 \%)$, para validação de ensaios considerados no processo de registro de novas cultivares. Poucos ensaios mostraram CV superiores a $20 \%$, relativos a esse caráter.

O limite máximo de $\mathrm{CV}$ aceitável em relação à produtividade, ou seja, considerados médios ou baixos, é de $22 \%$ no milho (Scapim et al., 1995) e 27,9\% no arroz de terras baixas (Costa et al., 2002). Na soja, esse limite foi de $16 \%$. Essa oscilação de valores foi também observada para altura da planta. No milho, o limite é de $13 \%$, no arroz de terras altas, de $7,4 \%$ e na soja, de $12 \%$ (Tabela 2). Assim, além do caráter, outro fator que deve ser considerado na avaliação da precisão experimental é a cultura estudada.

Tabela 4. Freqüência dos coeficientes de variação de produtividade e altura da planta de soja considerados baixo, médio, alto e muito alto, de acordo com os critérios de classificação de Garcia (1989), de Costa et al. (2002) e de Pimentel-Gomes (1985), no Paraná e Mato Grosso, em razão do grupo de maturação (precoce, semiprecoce e médio).

\begin{tabular}{|c|c|c|c|c|c|c|}
\hline \multirow{2}{*}{$\begin{array}{l}\text { Classificação } \\
\text { dos coeficientes } \\
\text { de variação }\end{array}$} & \multicolumn{3}{|c|}{ Produtividade } & \multicolumn{3}{|c|}{ Altura da planta } \\
\hline & Garcia (1989) & Costa et al. (2002) & Pimentel-Gomes (1985) & Garcia (1989) & Costa et al. (2002) & Pimentel-Gomes (1985) \\
\hline & \multicolumn{6}{|c|}{ Paraná - Precoce } \\
\hline Baixo & 11,9 & 12,5 & 38,09 & 13,2 & 13,2 & 82,45 \\
\hline Médio & 74,4 & 69,6 & 60,12 & 73,7 & 71,0 & 17,54 \\
\hline Alto & 8,9 & 9,5 & 1,79 & 7,0 & 7,9 & 0,00 \\
\hline \multirow[t]{2}{*}{ Muito alto } & 4,8 & 8,3 & 0,00 & 6,1 & 7,9 & 0,00 \\
\hline & \multicolumn{6}{|c|}{ Paraná - Semiprecoce } \\
\hline Baixo & 11,2 & 10,5 & 34,70 & 16,2 & 16,2 & 91,47 \\
\hline Médio & 72,4 & 68,4 & 61,77 & 69,8 & 66,9 & 8,53 \\
\hline Alto & 10,6 & 12,9 & 3,53 & 10,1 & 11,5 & 0,00 \\
\hline \multirow[t]{2}{*}{ Muito alto } & 5,8 & 8,2 & 0,00 & 3,9 & 5,4 & 0,00 \\
\hline & \multicolumn{6}{|c|}{ Paraná - Médio } \\
\hline Baixo & 12,2 & 12,3 & 28,66 & 12,5 & 14,2 & 82,79 \\
\hline Médio & 71,5 & 69,6 & 67,83 & 70,0 & 67,5 & 17,21 \\
\hline Alto & 12,2 & 11,1 & 3,51 & 10,0 & 10,0 & 0,00 \\
\hline \multirow[t]{2}{*}{ Muito alto } & 4,1 & 7,0 & 0,00 & 7,5 & 8,3 & 0,00 \\
\hline & \multicolumn{6}{|c|}{ Mato Grosso - Precoce } \\
\hline Baixo & 16,5 & 17,7 & 17,72 & 8,9 & 6,3 & 65,82 \\
\hline Médio & 69,5 & 68,4 & 81,01 & 70,8 & 73,4 & 34,18 \\
\hline Alto & 8,9 & 10,1 & 1,27 & 16,5 & 16,5 & 0,00 \\
\hline \multirow[t]{2}{*}{ Muito alto } & 5,1 & 3,8 & 0,00 & 3,8 & 3,8 & 0,00 \\
\hline & \multicolumn{6}{|c|}{ Mato Grosso - Médio } \\
\hline Baixo & 20,0 & 18,8 & 23,75 & 19,8 & 13,6 & 69,14 \\
\hline Médio & 60,0 & 61,2 & 76,25 & 64,2 & 71,6 & 30,86 \\
\hline Alto & 17,5 & 12,5 & 0,00 & 11,1 & 12,3 & 0,00 \\
\hline \multirow[t]{2}{*}{ Muito alto } & 2,5 & 7,5 & 0,00 & 4,9 & 2,5 & 0,00 \\
\hline & \multicolumn{6}{|c|}{ Mato Grosso - Tardio } \\
\hline Baixo & 17,3 & 20,9 & 20,99 & 17,7 & 11,4 & 68,35 \\
\hline Médio & 67,9 & 60,6 & 77,77 & 68,4 & 74,7 & 31,65 \\
\hline Alto & 12,3 & 13,6 & 1,24 & 11,4 & 11,4 & 0,00 \\
\hline Muito alto & 2,5 & 4,9 & 0,00 & 2,5 & 2,5 & 0,00 \\
\hline
\end{tabular}




\section{Conclusões}

1. O limite máximo do coeficiente de variação aceitável em relação à produtividade e à altura da planta de soja é de $16 \%$ e $12 \%$, respectivamente.

2. A classificação dos coeficientes de variação de ensaio experimental de soja, nos estados do Paraná e Mato Grosso, depende do caráter avaliado e da localização, mas oscila pouco em razão do ciclo reprodutivo.

3. Independentemente da distribuição, não há muita diferença na definição dos intervalos de classificação dos coeficientes de variação de produtividade e altura da planta de soja, ao se utilizar a mediana e pseudo-sigma, em substituição à média e desvio-padrão, respectivamente.

4. A classificação dos coeficientes de variação, em razão da média (ou mediana) e do desvio-padrão (ou pseudo-sigma) dos coeficientes, mostra-se satisfatória na avaliação da precisão experimental de ensaios de soja.

\section{Referências}

BRASIL. Ministério da Agricultura e do Abastecimento. Requisitos mínimos para determinação do valor de cultivo e uso de soja (Glycine max) para inscrição no re- gistro nacional de cultivares - RNC. Disponível em: <http://www.agricultura.gov.br/snpc/form1600.htm>. Acesso em: 21 out. 2001.

COSTA, N. H. de A. D.; SERAPHIN, J. C.; ZIMMERMANN, F. J. P. Novo método de classificação de coeficientes de variação para a cultura do arroz de terras altas. Pesquisa Agropecuária Brasileira, Brasília, v. 37, n. 3, p. 243-249, mar. 2002.

GARCIA, C. H. Tabelas para classificação do coeficiente de variação. Piracicaba: Instituto de Pesquisas e Estudos Florestais, 1989. 12 p. (Circular Técnica, 171).

PIMENTEL-GOMES, F. Curso de estatística experimental. São Paulo: Esalq, 1985. 467 p.

HOAGLIN, D. C.; MOSTELLER, F.; TUKEY, J. W. Understanding robust and exploratory data analysis. New York: J. Wiley, 1983. 447 p.

SAS INSTITUTE (Cary, Estados Unidos). SAS/STAT user's guide: statistics. 5th ed. Cary, 1990. 1686 p.

SCAPIM, C. A. S.; CARVALHO, C. G. P. de; CRUZ, C. D. Uma proposta de classificação dos coeficientes de variação para a cultura do milho. Pesquisa Agropecuária Brasileira, Brasília, v. 30, n. 5, p. 683-686, maio 1995.

TUKEY, J. W. Exploratory data analysis. Reading: Addison-Wesley, 1977. 688 p. 